\title{
PROJECTION ESTIMATES FOR MUTUAL MULTIFRACTAL DIMENSIONS
}

\section{BILEL SELMI}

Department of Mathematics

Faculty of Sciences of Monastir

Analysis, Probability \& Fractals Laboratory LR18ES17

5000-Monastir

Tunisia

e-mail: bilel.selmi@fsm.rnu.tn

\begin{abstract}
We study the mutual multifractal spectrum of orthogonal projections of a couple of measures $(\mu, \nu)$ having a finite $s$-energy for some $1 \leq m \leq s<n$.
\end{abstract}

\section{Introduction and Preliminaries}

Recently the projection behaviour of dimensions and multifractal spectra of measures has generated an interest in the mathematical literature $[8,18-23,25-28,34,39,41,43,45]$. This is connected to the question of the relationship between the Hausdorff and packing dimensions of a subset of $\mathbb{R}^{n}$ or a Borel probability measure and that of its orthogonal projections onto an $m$-dimensional subspace. The fundamental result on projections concerns Hausdorff dimensions: 2020 Mathematics Subject Classification: 28A80, 28A75, 26A18.

Keywords and phrases: Hausdorff dimension, packing dimension, mutual multifractal analysis, projection.

Received March 5, 2020; Revised May 20, 2020

(C) 2020 Scientific Advances Publishers 
the projection of an analytic subset $E$ of $\mathbb{R}^{n}$ onto almost all $m$-dimensional subspaces has a Hausdorff dimension equal to $\min \left(m, \operatorname{dim}_{H}(E)\right)$, where $\operatorname{dim}_{H}(E)$ denotes the Hausdorff dimension of $E$. This was proved by Marstrand [30], also in [29], Kaufman employed potential theoretic methods in order to prove Marstrand result's, which has been generalized later by Mattila in [31] and $\mathrm{Hu}$ and Taylor [26]. The behaviour of the packing dimension under projections is not as straightforward as that of the Hausdorff dimension. While the Hausdorff dimension of a set or a measure is preserved under almost all projections, its packing dimension may decrease for almost all of them [21, 22]. O'Neil and Selmi [34, 44] compared the generalized Hausdorff and packing dimensions of a set $E$ of $\mathbb{R}^{n}$ with respect to a measure $\mu$ with those of projections onto $m$-dimensional subspaces. The results of O'Neil were later generalized by Selmi et al. in [18, 41, 43]. In [19, 20, 45], the authors studied the mutual multifractal analysis (see [32, 46-49]) of the orthogonal projections on $m$-dimensional linear subspaces, more specifically, they investigated the relationship between $f_{\mu, \nu}(\alpha, \beta)$ and $f_{\mu_{V, \nu}}(\alpha, \beta)$, where

$$
\begin{gathered}
f_{\mu, \nu}(\alpha, \beta)=\operatorname{dim}_{K}\left(E_{\mu, \nu}(\alpha, \beta)\right), \\
E_{\mu, \nu}(\alpha, \beta)=\left\{\begin{array}{r}
x \in \operatorname{supp} \mu \cap \operatorname{supp} \nu ; \lim _{r \rightarrow 0} \frac{\log \mu(B(x, r))}{\log r}=\alpha \\
\text { and } \lim _{r \rightarrow 0} \frac{\log \nu(B(x, r))}{\log r}=\beta
\end{array}\right\},
\end{gathered}
$$

and $K \in\{H, P\}$, here $\operatorname{dim}_{P}$ denotes the packing dimension. Recently, there has been a great interest in this subject (the calculus of $f_{\mu, \nu}(\alpha, \beta)$ ) and positive results have been written in various situations in the dynamic contexts [3-7, 37]. Lately, many authors focused on mutual (mixed) multifractal spectra, see, for example, [1, 2, 9, 10, 12-17, 32, 33, 36, 38]. 
As a continuity of these researches, we investigate the mutual multifractal spectrum of orthogonal projections of a couple of measures $(\mu, \nu)$ having a finite $s$-energy (see (2.1) for the definition).

Casually, we briefly recall some basic definitions and facts which will be repeatedly used in subsequent developments. For an arbitrary Borel probability measures $\mu$ and $\nu$ on $\mathbb{R}^{n}$, they introduced two threeparameter families of measures, $\quad\left\{\mathcal{P}_{\mu, \nu}^{q, t, s} ; q, t, s \in \mathbb{R}\right\} \quad$ and $\left\{\mathcal{H}_{\mu, \nu}^{q, t, s} ; q, t, s \in \mathbb{R}\right\}$ based on certain generalizations of the Hausdorff measure and of the packing measure. For $q, t, s \in \mathbb{R}, E \subseteq \mathbb{R}^{n}$ and $\delta>0$, we define

$$
\overline{\mathcal{P}}_{\mu, \nu, \delta}^{q, t, s}(E)=\sup \left\{\begin{array}{r}
\sum_{i} \mu\left(B_{i}\right)^{q} \nu\left(B_{i}\right)^{t}\left(2 r_{i}\right)^{s} ;\left(B_{i}=B\left(x_{i}, r_{i}\right)\right)_{i} \\
\text { is a centered } \delta \text {-packing of } E
\end{array}\right\},
$$

and

$$
\overline{\mathcal{P}}_{\mu, \nu}^{q, t, s}(E)=\inf _{\delta>0} \overline{\mathcal{P}}_{\mu, \nu, \delta}^{q, t, s}(E)
$$

The mutual packing measure is then given by

$$
\mathcal{P}_{\mu, \nu}^{q, t, s}(E)=\inf _{E \subseteq \bigcup_{i} E_{i}} \sum_{i} \overline{\mathcal{P}}_{\mu, \nu}^{q, t, s}\left(E_{i}\right) .
$$

In a similar way, we define

$$
\overline{\mathcal{H}}_{\mu, \nu, \delta}^{q, t, s}(E)=\inf \left\{\begin{array}{r}
\sum_{i} \mu\left(B_{i}\right)^{q} \nu\left(B_{i}\right)^{t}\left(2 r_{i}\right)^{s} ;\left(B_{i}=B\left(x_{i}, r_{i}\right)\right)_{i} \\
\text { is a centered } \delta \text {-cover of } E
\end{array}\right\},
$$

and

$$
\overline{\mathcal{H}}_{\mu, \nu}^{q, t, s}(E)=\sup _{\delta>0} \overline{\mathcal{H}}_{\mu, \nu, \delta}^{q, t, s}(E)
$$


The mutual Hausdorff measure is defined by

$$
\mathcal{H}_{\mu, \nu}^{q, t, s}(E)=\sup _{F \subseteq E} \overline{\mathcal{H}}_{\mu, \nu}^{q, t, s}(F)
$$

The measures $\mathcal{H}_{\mu, \nu}^{q, t, s}$ and $\mathcal{P}_{\mu, \nu}^{q, t, s}$ and the pre-measure $\overline{\mathcal{P}}_{\mu, \nu}^{q, t, s}$ assign, in the usual way, a mutual multifractal dimension to each subset $E$ of $\mathbb{R}^{n}$. They are respectively denoted by $\operatorname{dim}_{\mu, \nu}^{q, t}(E), \operatorname{Dim}_{\mu, \nu}^{q, t}(E)$, and $\Delta_{\mu, \nu}^{q, t}(E)$ (see $[46,48]$ ). More precisely, we have

$$
\begin{aligned}
& b_{\mu, \nu}^{q, t}(E)=\inf \left\{s \in \mathbb{R}, \quad \mathcal{H}_{\mu, \nu}^{q, t, s}(E)=0\right\}, \\
& B_{\mu, \nu}^{q, t}(E)=\inf \left\{s \in \mathbb{R}, \quad \mathcal{P}_{\mu, \nu}^{q, t, s}(E)=0\right\}, \\
& \Delta_{\mu, \nu}^{q, t}(E)=\inf \left\{s \in \mathbb{R}, \quad \overline{\mathcal{P}}_{\mu, \nu}^{q, t, s}(E)=0\right\} .
\end{aligned}
$$

It is clear that

$$
b_{\mu, \nu}^{q, t}(E) \leq B_{\mu, \nu}^{q, t}(E) \leq \Delta_{\mu, \nu}^{q, t}(E)
$$

Next we define the mutual multifractal dimension functions $b_{\mu, \nu}, B_{\mu, \nu}$ and $\Lambda_{\mu, \nu}: \mathbb{R}^{2} \rightarrow[-\infty,+\infty]$ by

$$
\begin{gathered}
b_{\mu, \nu}:(q, t) \rightarrow b_{\mu, \nu}^{q, t}(\operatorname{supp} \mu \cap \operatorname{supp} \nu), \\
B_{\mu, \nu}:(q, t) \rightarrow B_{\mu, \nu}^{q, t}(\operatorname{supp} \mu \cap \operatorname{supp} \nu), \\
\Lambda_{\mu, \nu}:(q, t) \rightarrow \Delta_{\mu, \nu}^{q, t}(\operatorname{supp} \mu \cap \operatorname{supp} \nu) .
\end{gathered}
$$

\section{Remark 1.1.}

(1) The measure $\mathcal{H}_{\mu, \nu}^{q, t, s}$ is a multifractal generalisation of the centered Hausdorff measure, whereas $\mathcal{P}_{\mu, \nu}^{q, t, s}$ is a multifractal generalisation of the packing measure. In fact, it is easily seen that if $s \geq 0$, then $\mathcal{H}_{\mu, \nu}^{0,0, s}=\mathcal{H}^{s}$ 
and $\mathcal{P}_{\mu, \nu}^{0,0, s}=\mathcal{P}^{s}$, where $\mathcal{H}^{s}$ denotes the $s$-dimensional centered Hausdorff measure and $\mathcal{P}^{s}$ denotes the $s$-dimensional packing measure (see [35] for more information on $\mathcal{H}^{s}$ and $\mathcal{P}^{s}$ ).

(2) In the special case where $q=0$ or $t=0$, the mutual multifractal spectra is strictly related to Olsen's multifractal formalism [35].

(3) The mutual multifractal spectra represents the relative multifractal analysis introduced by Cole [11] (see also [40, 42]) in the case where $s=0$.

\section{The Main Results}

We denote by $G_{n, m}$ the Grassmannian manifold of all $m$-dimensional linear subspaces of $\mathbb{R}^{n}$ and $\gamma_{n, m}$ its orthogonally invariant Borel probability measure. We write $\mathcal{L}^{n}$ to denote $n$-dimensional Lebesgue measure on any $n$-dimensional plane. We denote by $\pi_{V}$ the orthogonal projection onto a linear subspace $V$ of $\mathbb{R}^{n}$. Now, for a Borel probability measure $\mu$ on $\mathbb{R}^{n}$, supported on the compact set supp $\mu$ and for $V \in G_{n, m}$ we define $\mu_{V}$, the projection of $\mu$ onto $V$ by

$$
\mu_{V}(A)=\mu\left(\pi_{V}^{-1}(A)\right) \quad \forall A \subseteq V
$$

Since $\mu$ has a compact support, $\operatorname{supp} \mu_{V}=\pi_{V}(\operatorname{supp} \mu)$ for all $V \in G_{n, m}$, then for any continuous function $f: V \longrightarrow \mathbb{R}$

$$
\int_{V} f d \mu_{V}=\int f\left(\pi_{V}(x)\right) d \mu(x)
$$

whenever these integrals exist. For $s$ an integer with $1 \leq m \leq s<n$, we denote the $s$-energy of a measure $\mu$ by

$$
I_{s}(\mu)=\iint|x-y|^{-s} d \mu(x) d \mu(y)
$$


Frostman [24] showed that the Hausdorff dimension of a Borel subset $E$ of $\mathbb{R}^{n}$ is the supremum of the positive reals $s$ for which there exists a Borel probability measure $\mu$ charging $E$ and for which the $s$-energy of $\mu$ is finite. This characterization is used by Kaufman [29] and Mattila [31] to prove their results on the preservation of the Hausdorff dimension. The conditions : $I_{s}(\mu)$ is finite, implies that $\operatorname{dim}_{H}(\mu) \geq s$, on the other hand if $\mu(B(x, r)) \leq r^{s}$ for all $x$ and all sufficiently small $r$, then $\mu$ has a finite $s$-energy. Notice Mattila [31] proved that if $I_{m}(\mu)$ is finite, then for almost every $m$-dimensional subspaces $V$, measure $\mu_{V}$ is absolutely continuous with respect to Lebesgue measure $\mathcal{L}_{V}^{m}$ on $V$ identified with $\mathbb{R}^{m}$, where $\mathcal{L}_{V}^{m}(E)=\mathcal{L}^{m}(E \cap V)$ for $E \subset \mathbb{R}^{m}$, and $\mu_{V} \in L^{2}(V)$.

Throughout this paper, we suppose that $\mu$ and $\nu$ be two compactly supported Borel probability measures with $\operatorname{supp} \mu=\operatorname{supp} \nu=K$. Our main results are the following: in the next few results, we describe how if the couple of measures $(\mu, \nu)$ having a finite $s$-energy for some $s>m$, then we may deduce a little more about the usual structure of the projections of measures.

Theorem 2.1. Let $1 \leq m \leq s<n$, suppose that $I_{s}(\mu)<\infty$ and $I_{s}(\nu)<\infty$. Then for almost every $m$-dimensional subspace $V$ and all $t, q \geq 1$, we have

(1) If $m<s<2 m$, then

$$
\begin{aligned}
& m(1-(q+t)) \leq b_{\mu_{V}, \nu_{V}}(q, t) \leq B_{\mu_{V}, \nu_{V}}(q, t) \leq \Lambda_{\mu_{V}, \nu_{V}}(q, t) \\
& \quad \leq \max \left\{-\frac{s(q+t)}{2}, m(2-(q+t)),-\frac{s t}{2}+m(1-q),-\frac{s q}{2}+m(1-t)\right\} .
\end{aligned}
$$

(2) If $s \geq 2 m$, then

$$
\begin{aligned}
m(1-(q+t)) & \leq b_{\mu_{V}, \nu_{V}}(q, t) \leq B_{\mu_{V}, \nu_{V}}(q, t) \leq \Lambda_{\mu_{V}, \nu_{V}}(q, t) \\
& \leq m(2-(q+t)) .
\end{aligned}
$$


Definition 2.1. We say that two Borel measures $\mu$ and $\nu$ are equivalent and we write $\nu \sharp \mu$ if there exists $c>1$ such that for a Borel set $A$ in $\mathbb{R}^{n}$,

$$
c^{-1} \mu_{\left\lfloor_{\perp} \operatorname{supp} \nu\right.}(A) \leq \nu(A) \leq c \mu_{\lfloor\operatorname{supp} \nu}(A)
$$

Theorem 2.2. Let $1 \leq m \leq s<n$, we assume that $I_{s}(\mu)<\infty$ and $\nu \sharp \mathcal{L}^{n}$. Then for almost every m-dimensional subspace $V$ and all $(q, t) \in[1,+\infty[\times \mathbb{R}$, we have

(1) If $m<s<2 m$, then

(a) For $q \geq \frac{2 m}{2 m-s}$

$$
\begin{aligned}
m(1-(q+t)) \leq b_{\mu_{V}, \nu_{V}}(q, t) & \leq B_{\mu_{V}, \nu_{V}}(q, t) \\
& \leq \Lambda_{\mu_{V}, \nu_{V}}(q, t) \leq-\left(\frac{s q}{2}+m t\right) .
\end{aligned}
$$

(b) For $1 \leq q \leq \frac{2 m}{2 m-s}$

$$
b_{\mu_{V}, \nu_{V}}(q, t)=B_{\mu_{V}, \nu_{V}}(q, t)=\Lambda_{\mu_{V}, \nu_{V}}(q, t)=m(1-(q+t)) .
$$

(2) If $s \geq 2 m$, then

$$
b_{\mu_{V}, \nu_{V}}(q, t)=B_{\mu_{V}, \nu_{V}}(q, t)=\Lambda_{\mu_{V}, \nu_{V}}(q, t)=m(1-(q+t)) .
$$

The symmetrical results are true as well.

Theorem 2.3. Let $1 \leq m \leq s<n$, we suppose that $\mu \sharp \mathcal{L}^{n}$ and $I_{s}(\nu)<\infty$. Then for almost every m-dimensional subspace $V$ and all $(q, t) \in \mathbb{R} \times[1,+\infty[$, we have 
(1) If $m<s<2 m$, then

(a) For $t \geq \frac{2 m}{2 m-s}$

$$
\begin{aligned}
m(1-(q+t)) \leq b_{\mu_{V}, \nu_{V}}(q, t) & \leq B_{\mu_{V}, \nu_{V}}(q, t) \\
& \leq \Lambda_{\mu_{V}, \nu_{V}}(q, t) \leq-\left(\frac{s t}{2}+m q\right) .
\end{aligned}
$$

(b) For $1 \leq t \leq \frac{2 m}{2 m-s}$

$$
b_{\mu_{V}, \nu_{V}}(q, t)=B_{\mu_{V}, \nu_{V}}(q, t)=\Lambda_{\mu_{V}, \nu_{V}}(q, t)=m(1-(q+t)) .
$$

(2) If $s \geq 2 m$, then

$$
b_{\mu_{V}, \nu_{V}}(q, t)=B_{\mu_{V}, \nu_{V}}(q, t)=\Lambda_{\mu_{V}, \nu_{V}}(q, t)=m(1-(q+t)) .
$$

\section{Remark 2.1.}

(1) We assume that $\mu$ and $\nu$ be two Borel probability measures having the same compact support with $\mu \sharp \mathcal{L}^{n}$ and $\nu \sharp \mathcal{L}^{n}$. Then for all $m$-dimensional subspace $V$ and all $(t, q) \in \mathbb{R}^{2}$, we have

$$
b_{\mu_{V}, \nu_{V}}(q, t)=B_{\mu_{V}, \nu_{V}}(q, t)=\Lambda_{\mu_{V}, \nu_{V}}(q, t)=m(1-(q+t)) .
$$

(2) The results developed by O'Neil in [34] are obtained as a special case of the mutual multifractal theorems by setting $q=0$ or $t=0$.

Example 2.1. The hypothesis $\operatorname{supp} \mu=\operatorname{supp} \nu$ is sufficient to obtain the conclusion (2.2). In fact, considering a special case where $(q, t)=(0,0)$, we can find two measures $\mu$ and $\nu$ such that $\operatorname{supp} \mu \subset \operatorname{supp} \nu$ and

$$
b_{\mu_{V}, \nu_{V}}(q, t)<b_{\mu, \nu}(q, t)<m(1-(q+t)) .
$$


Let $\mathcal{C}$ be the usual Cantor subset of $[0,1]$. Also, let $\mathcal{H}^{s}$ be the normalized $s=\frac{\log 2}{\log 3}$-dimensional Hausdorff measure on $\mathcal{C}$ and write

$$
\mu=\mathcal{H}^{s} \times \delta_{0},
$$

where $\delta_{0}$ denotes the Dirac measure concentrated at 0 and

$$
K=\operatorname{supp} \mu=\mathcal{C} \times\{0\}
$$

Taking $\nu$ to be the normalized 2-dimensional Lebesgue measure on a ball with center at $(0,0)$ and radius equal to 2 , it is clear that $\operatorname{supp} \mu \subset \operatorname{supp} \nu$. We note that for any 1-dimensional subspace $V$ of $\mathbb{R}^{2}$, we have

$$
b_{\mu, \nu}(0,0)=\operatorname{dim}_{H}(\operatorname{supp} \mu)=\operatorname{dim}_{H}(K)=s,
$$

and

$$
b_{\mu_{V}, \nu_{V}}(0,0)=\operatorname{dim}_{H}\left(\operatorname{supp} \mu_{V}\right)=\operatorname{dim}_{H}\left(K_{V}\right),
$$

where $K_{V}$ denotes the projection of $K$ onto $V$. For $V=\{0\} \times \mathbb{R}$ we have $K_{V}=\{(0,0)\}$, whence

$$
b_{\mu_{V}, \nu_{V}}(0,0)=\operatorname{dim}_{H}\left(K_{V}\right)=0<s=\operatorname{dim}_{H}(K)=b_{\mu, \nu}(0,0)<1 .
$$

\section{Proof of Main Results}

Before proving the main results we need some preliminary results. We begin by investigating the multifractal spectrum of a measure which is absolutely continuous with respect to Lebesgue measure.

Proposition 3.1. Suppose that $\mu$ and $\nu$ are absolutely continuous with respect to Lebesgue measure on $K$. Then for $q, t \geq 0$

$$
b_{\mu, \nu}(q, t) \geq n(1-(q+t)) .
$$


Proof. Suppose that $f \geq 0$ is such that $\mu=f \mathcal{L}_{K}^{n}$. Then, as $\mu(K)>0$ we can find a Borel set $A \subset K$ of positive Lebesgue measure and $\gamma_{1}>0$ such that for all $x \in A, f(x) \geq \gamma_{1}$. Similarly, we suppose the same for $g$, i.e., there exist a Borel set $B \subset K$ of positive Lebesgue measure and $\gamma_{2}>0$ such that for all $x \in B, g(x) \geq \gamma_{2}$, where $g \geq 0$ such that $\nu=g \mathcal{L}_{K}^{n}$. Let $\left(B_{i}=B\left(x_{i}, r_{i}\right)\right)_{i}$ be a centered $\delta$-covering of $K$. For $s<n(1-(q+t))$, we obtain

$$
\begin{aligned}
\sum_{i} \mu\left(B_{i}\right)^{q} \nu\left(B_{i}\right)^{t}\left(2 r_{i}\right)^{s} & \geq 2^{s} \sum_{i}\left(\int_{B_{i}} f d \mathcal{L}^{n}\right)^{q}\left(\int_{B_{i}} g d \mathcal{L}^{n}\right)^{t} r_{i}^{s} \\
& \geq 2^{s} \gamma_{1}^{q} \gamma_{2}^{t} \sum_{i} \mathcal{L}^{n}\left(A \cap B_{i}\right)^{q} \mathcal{L}^{n}\left(B \cap B_{i}\right)^{t} r_{i}^{s} \\
& \geq 2^{s} \alpha(n)^{-\frac{s}{n}} \gamma_{1}^{q} \gamma_{2}^{t} \sum_{i} \sup \left\{\mathcal{L}_{A}^{n}\left(B_{i}\right)^{q+t+\frac{s}{n}}, \mathcal{L}_{B}^{n}\left(B_{i}\right)^{q+t+\frac{s}{n}}\right\} \\
& \geq 2^{s} \alpha(n)^{-\frac{s}{n}} \gamma_{1}^{q} \gamma_{2}^{t} \sup \left\{\mathcal{L}^{n}(A)^{q+t+\frac{s}{n}}, \mathcal{L}^{n}(B)^{q+t+\frac{s}{n}}\right\} \\
& >0,
\end{aligned}
$$

where $\alpha(n)$ denotes the Lebesgue measure of the unit ball in $\mathbb{R}^{n}$. Hence $\mathcal{H}_{\mu, \nu}^{q, t, s}(K)>0$ which implies that $b_{\mu, \nu}(q, t) \geq s$ and the inequality follows.

Definition 3.1. For a measure $\mu$ on $\mathbb{R}^{n}$ we say, for $p \geq 1$, that $\mu \in L^{p}\left(\mathbb{R}^{n}\right)$ if there is a function $f \in L^{p}\left(\mathbb{R}^{n}\right)$ such that $f$ is the RadonNikodym derivative of $\mu$ with respect to $\mathcal{L}^{n}$ for $\mu$-a.e. $x$. 
Proposition 3.2. Fix $p \in\left[1,+\infty\left[\right.\right.$. Suppose that $\mu, \nu \in L^{p}\left(\mathbb{R}^{n}\right)$. Then for $q, t \geq 1$,

$$
\begin{aligned}
\Lambda_{\mu, \nu}(q, t) \leq & \max \left\{n \frac{(q+t)(1-p)}{p}, n(2-(q+t)),\right. \\
& \left.n\left(\frac{t(1-p)}{p}+1-q\right), n\left(\frac{q(1-p)}{p}+1-t\right)\right\} .
\end{aligned}
$$

Proof. Since $\mu, \nu \in L^{p}\left(\mathbb{R}^{n}\right)$, there exists a compactly supported function $f$ (resp., $g$ ) on $K$ such that $f$ (resp., $g$ ) is the Radon-Nikodym derivative of $\mu$ (resp., $\nu$ ) with respect to $\mathcal{L}^{n}$ for $\mu$ (resp., $\nu$ )-a.e. $x$.

We distinguish four different cases.

- Case 1: For $t, q \geq p \geq 1$.

Let $s>-n(q+t) \frac{p-1}{p}, \delta>0$ and $\left(B_{i}=B\left(x_{i}, r_{i}\right)\right)_{i}$ be a centered $\delta$-packing of $K$. By using Hölder's inequality, we obtain

$$
\begin{aligned}
& \sum_{i} \mu\left(B_{i}\right)^{q} \nu\left(B_{i}\right)^{t}\left(2 r_{i}\right)^{s} \\
& \leq 2^{s} \sum_{i}\left(\int_{B_{i}} f d \mathcal{L}^{n}\right)^{q}\left(\int_{B_{i}} g d \mathcal{L}^{n}\right)^{t} r_{i}^{s} \\
& \leq 2^{s} \alpha(n)^{-\frac{s}{n}} \sum_{i}\left(\int_{B_{i}} f d \mathcal{L}^{n}\right)^{q}\left(\int_{B_{i}} g d \mathcal{L}^{n}\right)^{t} \mathcal{L}^{n}\left(B_{i}\right)^{\frac{s}{n}} \\
& \leq 2^{s} \alpha(n)^{-\frac{s}{n}} \sum_{i}\left[\left(\int_{B_{i}} g^{p} d \mathcal{L}^{n}\right)^{\frac{q}{p}}\left(\int_{B_{i}} g^{p} d \mathcal{L}^{n}\right)^{\frac{t}{p}} \times \mathcal{L}^{n}\left(B_{i}\right)^{\frac{s}{n}} \mathcal{L}^{n}\left(B_{i}\right)^{\left(\frac{q(p-1)}{P}\right)} \mathcal{L}^{n}\left(B_{i}\right)^{\left(\frac{t(p-1)}{P}\right)}\right] \\
& \leq 2^{s} \alpha(n)^{-\frac{s}{n}} \sum_{i}\left(\int_{B_{i}} f^{p} d \mathcal{L}^{n}\right)^{\frac{q}{p}}\left(\int_{B_{i}} g^{p} d \mathcal{L}^{n}\right)^{\frac{t}{p}} \mathcal{L}^{n}\left(B_{i}\right)^{\frac{s}{n}+(q+t) \frac{p-1}{p}}
\end{aligned}
$$




$$
\begin{aligned}
& \leq 2^{s} \alpha(n)^{-\frac{s}{n}} \sum_{i}\left(\int_{B_{i}} f^{p} d \mathcal{L}^{n}\right)^{\frac{q}{p}}\left(\int_{B_{i}} g{ }^{p} d \mathcal{L}^{n}\right)^{\frac{t}{p}} \\
& \leq 2^{s} \alpha(n)^{-\frac{s}{n}} \sum_{i}\left(\int_{B_{i}} f^{p} d \mathcal{L}^{n}\right)^{\frac{q}{p}} \sum_{i}\left(\int_{B_{i}} g^{p} d \mathcal{L}^{n}\right)^{\frac{t}{p}} \\
& \leq 2^{s} \alpha(n)^{-\frac{s}{n}}\left(\sum_{i} \int_{B_{i}} f^{p} d \mathcal{L}^{n}\right)^{\frac{q}{p}}\left(\sum_{i} \int_{B_{i}} g{ }^{p} d \mathcal{L}^{n}\right)^{\frac{t}{p}} \\
& =2^{s} \alpha(n)^{-\frac{s}{n}}\left(\int f^{p} d \mathcal{L}^{n}\right)^{\frac{q}{p}}\left(\int g^{p} d \mathcal{L}^{n}\right)^{\frac{t}{p}}<\infty,
\end{aligned}
$$

where $\alpha(n)$ denotes the Lebesgue measure of the unit ball in $\mathbb{R}^{n}$.

- Case 2: For $p \geq t \geq 1$ and $p \geq q \geq 1$.

Suppose that $s>-n(q+t-2)$. Let $\delta>0$ and $\left(B_{i}=B\left(x_{i}, r_{i}\right)\right)_{i}$ be a centered $\delta$-packing of $K$. Then we find, on using Hölder's inequality that

$$
\begin{aligned}
\sum_{i} \mu\left(B_{i}\right)^{q} \nu\left(B_{i}\right)^{t}\left(2 r_{i}\right)^{s} & \\
& \leq 2^{s} \sum_{i}\left(\int_{B_{i} \cap K} f d \mathcal{L}^{n}\right)^{q}\left(\int_{B_{i} \cap K} g d \mathcal{L}^{n}\right)^{t} r_{i}^{s} \\
& \leq 2^{s} \alpha(n)^{-\frac{s}{n}} \sum_{i}\left(\int_{B_{i} \cap K} f^{q} d \mathcal{L}^{n}\right)\left(\int_{B_{i} \cap K} g^{t} d \mathcal{L}^{n}\right) \mathcal{L}_{K}^{n}\left(B_{i}\right)^{q+t-2+\frac{s}{n}} \\
& \leq 2^{s} \alpha(n)^{-\frac{s}{n}} \sum_{i}\left(\int_{B_{i} \cap K} f^{q} d \mathcal{L}^{n}\right) \sum_{i}\left(\int_{B_{i} \cap K} g^{t} d \mathcal{L}^{n}\right) \\
& \leq 2^{s} \alpha(n)^{-\frac{s}{n}}\left(\int_{K} f^{q} d \mathcal{L}^{n}\right)\left(\int_{K} g^{t} d \mathcal{L}^{n}\right)<\infty
\end{aligned}
$$

where $\alpha(n)$ denotes the Lebesgue measure of the unit ball in $\mathbb{R}^{n}$. 
- Case 3: For $t \geq p \geq q \geq 1$.

Fix $\quad s>-n\left(\frac{t(p-1)}{p}+q-1\right), \delta>0 \quad$ and $\quad\left(B_{i}=B\left(x_{i}, r_{i}\right)\right)_{i} \quad$ be $\quad$ a centered $\delta$-packing of $K$. Hölder's inequality gives

$$
\begin{aligned}
& \sum_{i} \mu\left(B_{i}\right)^{q} \nu\left(B_{i}\right)^{t}\left(2 r_{i}\right)^{s} \\
& \quad \leq 2^{s} \sum_{i}\left(\int_{B_{i} \cap K} f d \mathcal{L}^{n}\right)^{q}\left(\int_{B_{i} \cap K} g d \mathcal{L}^{n}\right)^{t} r_{i}^{s} \\
& \quad \leq 2^{s} \alpha(n)^{-\frac{s}{n}} \sum_{i}\left[\left(\int_{B_{i} \cap K} f^{q} d \mathcal{L}^{n}\right)\left(\int_{B_{i} \cap K} g^{p} d \mathcal{L}^{n}\right)^{\frac{t}{p}} \times \mathcal{L}^{n}\left(B_{i} \cap K\right)^{\left(\frac{t(p-1)}{P}+q-1+\frac{s}{n}\right)}\right] \\
& \quad \leq 2^{s} \alpha(n)^{-\frac{s}{n}} \sum_{i}\left(\int_{B_{i} \cap K} f^{q} d \mathcal{L}^{n}\right)\left(\int_{B_{i} \cap K} g{ }^{p} d \mathcal{L}^{n}\right)^{\frac{t}{p}} \\
& \quad \leq 2^{s} \alpha(n)^{-\frac{s}{n}} \sum_{i}\left(\int_{B_{i} \cap K} f^{q} d \mathcal{L}^{n}\right) \sum_{i}\left(\int_{B_{i} \cap K} g^{p} d \mathcal{L}^{n}\right)^{\frac{t}{p}} \\
& \quad \leq 2^{s} \alpha(n)^{-\frac{s}{n}}\left(\sum_{i} \int_{B_{i} \cap K} f^{q} d \mathcal{L}^{n}\right)\left(\sum_{i} \int_{B_{i} \cap K} g^{p} d \mathcal{L}^{n}\right)^{\frac{t}{p}} \\
& \leq 2^{s} \alpha(n)^{-\frac{s}{n}}\left(\int_{K} f^{q} d \mathcal{L}^{n}\right)\left(\int_{K} g{ }^{p} d \mathcal{L}^{n}\right)^{\frac{t}{p}}<\infty,
\end{aligned}
$$

where $\alpha(n)$ denotes the Lebesgue measure of the unit ball in $\mathbb{R}^{n}$. 
- Case 4: For $q \geq p \geq t \geq 1$. The proof of Case 4 is identical to the proof of the above case and is therefore omitted.

Finally, we obtain $\overline{\mathcal{P}}_{\mu, \nu}^{q, t, s}(K)<\infty$ which implies that $\Lambda_{\mu, \nu}(q, t) \leq s$ and the result follows.

We now describe conditions which ensure that a measure has projections which are in $L^{p}$ for some $p>1$.

Theorem 3.1 ([23]). Let $\mu$ be a compactly supported Radon measure on $\mathbb{R}^{n}$. Let $m \leq s<n$ and suppose that $I_{s}(\mu)<\infty$. Then $\mu_{V}$ is absolutely continuous with respect to $\mathcal{L}_{V}^{m}$, with $\mu_{V} \in L^{2}(V)$ for $\gamma_{n, m}$-almost all $V \in G_{n, m}$.

Moreover, for almost every m-dimensional subspace $V$,

(1) If $m<s<2 m$, then $\mu_{V} \in L^{p}(V)$ for all $p$ satisfying $1 \leq p<\frac{2 m}{2 m-s}$.

(2) If $2 m \leq s<n$, then the Radon-Nikodym derivation of $\mu_{V}$ with respect to $\mathcal{L}_{V}^{m}$ is bounded and essentially continuous.

Proof of Theorem 2.1. Since $I_{s}(\mu)<\infty$ and $I_{s}(\nu)<\infty$, it follows from Theorem 3.1 that, for almost every $m$-dimensional subspace $V$,

(1) If $m<s<2 m$, then $\mu_{V}, \nu_{V} \in L^{p}(V)$ for all $p$ satisfying $1 \leq p<\frac{2 m}{2 m-s}$.

(2) If $2 m \leq s<n$, then the Radon-Nikodym derivation of $\mu_{V}\left(\nu_{V}\right)$ with respect to $\mathcal{L}_{V}^{m}$ is bounded and essentially continuous.

Finally, the desired result follows directly from Propositions 3.1 and 3.2. 
Proof of Theorems 2.2 and 2.3. All of the ideas required to prove Theorems 2.2 and 2.3 can be found in the proof of Theorem 2.1.

\section{Acknowledgements}

The author is greatly indebted to the referee for his/her carefully reading the first submitted version of this paper and giving elaborate comments and valuable suggestions on revision so that the presentation can be greatly improved.

\section{References}

[1] M. Abid, M. Slimane, I. Omrane and B. Halouani, Mixed wavelet leaders multifractal formalism in a product of critical besov spaces, Mediterranean Journal of Mathematics 14(4) (2017), 1-20; Article 176.

DOI: https://doi.org/10.1007/s00009-017-0964-0

[2] J. Aouidi and A. Ben Mabrouk, A wavelet multifractal formalism for simultaneous singularities of functions, International Journal of Wavelets, Multiresolution and Information Processing 12(1) (2014); Article no. 1450009.

DOI: https://doi.org/10.1142/S021969131450009X

[3] L. Barreira, B. Saussol and J. Schmeling, Higher-dimensional multifractal analysis, Journal de Mathématiques Pures et Appliquées 81(4) (2002), 67-91.

DOI: https://doi.org/10.1016/S0021-7824(01)01228-4

[4] L. Barreira and P. Doutor, Birkhoff averages for hyperbolic flows: Variational principles and applications, Journal of Statistical Physics 115(5-6) (2004), 1567-1603.

DOI: https://doi.org/10.1023/B:JOSS.0000028069.64945.65

[5] L. Barreira and P. Doutor, Almost additive multifractal analysis, Journal de Mathématiques Pures et Appliquées 92(1) (2009), 1-17.

DOI: https://doi.org/10.1016/j.matpur.2009.04.006

[6] L. Barreira and P. Doutor, Dimension spectra of almost additive sequences, Nonlinearity 22(11) (2009), 2761-2773.

DOI: https://doi.org/10.1088/0951-7715/22/11/009

[7] L. Barreira, Y. Cao and J. Wang, Multifractal analysis of asymptotically additive sequences, Journal of Statistical Physics 153(5) (2013), 888-910.

DOI: https://doi.org/10.1007/s10955-013-0853-2 
[8] J. Barral and I. Bhouri, Multifractal analysis for projections of Gibbs and related measures, Ergodic Theory and Dynamic Systems 31(3) (2011), 673-701.

DOI: https://doi.org/10.1017/S0143385710000143

[9] M. Ben Slimane, Baire typical results for mixed Hölder spectra on product of continuous Besov or oscillation spaces, Mediterranean Journal of Mathematics 13(4) (2016), 1513-1533.

\section{DOI: https://doi.org/10.1007/s00009-015-0592-5}

[10] M. Ben Slimane, A. Ben Mabrouk and Jamil Aouidi, Mixed multifractal analysis for functions: General upper bound and optimal results for vectors of self-similar or quasi-self-similar of functions and their superpositions, Fractals 24(4) (2016); Article 1650039 .

DOI: https://doi.org/10.1142/S0218348X16500390

[11] J. Cole, Relative multifractal analysis, Chaos, Solitons \& Fractals 11(14) (2000), 2233-2250.

DOI: https://doi.org/10.1016/S0960-0779(99)00143-5

[12] M. Dai, Mixed self-conformal multifractal measures, Analysis in Theory and Applications 25(2) (2009), 154-165.

DOI: https://doi.org/10.1007/s10496-009-0154-4

[13] M. Dai and Y. Shi, Typical behavior of mixed $L^{q}$-dimensions, Nonlinear Analysis: Theory, Methods \& Applications 72(5) (2010), 2318-2325.

DOI: https://doi.org/10.1016/j.na.2009.10.032

[14] M. Dai and W. Li, The mixed $L^{q}$-spectra of self-conformal measures satisfying the weak separation condition, Journal of Mathematical Analysis and Applications 382(1) (2011), 140-147.

DOI: https://doi.org/10.1016/j.jmaa.2011.04.037

[15] C. Wang, H. Sun and M. Dai, Mixed generalized dimensions of random self-similar measures, International Journal of Nonlinear Science 13(1) (2012), 123-128.

[16] M. Dai, J. Hou, J. Gao, W. Su, L. Xi and D. Ye, Mixed multifractal analysis of China and US stock index series, Chaos, Solitons \& Fractals 87 (2016), 268-275.

DOI: https://doi.org/10.1016/j.chaos.2016.04.013

[17] M. Dai, S. Shao, J. Gao, Y. Sun and W. Su, Mixed multifractal analysis of crude oil, gold and exchange rate series, Fractals 24(1) (2016); Article 1650046.

DOI: https://doi.org/10.1142/S0218348X16500468

[18] Z. Douzi and B. Selmi, Multifractal variation for projections of measures, Chaos, Solitons \& Fractals 91 (2016), 414-420.

DOI: https://doi.org/10.1016/j.chaos.2016.06.026 
[19] Z. Douzi and B. Selmi, On the Projections of Mutual Multifractal Spectra, arXiv: $1805.06866 \mathrm{v} 1,2018$.

[20] Z. Douzi and B. Selmi, On the projections of the mutual multifractal Rényi dimensions, Analysis in Theory and Applications (to appear).

[21] K. J. Falconer and J. D. Howroyd, Packing dimensions of projections and dimensions profiles, Mathematical Proceedings of the Cambridge Philosophical Society 121(2) (1997), 269-286.

\section{DOI: https://doi.org/10.1017/S0305004196001375}

[22] K. J. Falconer and P. Mattila, The packing dimensions of projections and sections of measures, Mathematical Proceedings of the Cambridge Philosophical Society 119(4) (1996), 695-713.

DOI: https://doi.org/10.1017/S0305004100074533

[23] K. J. Falconer and T. C. O'Neil, Convolutions and the geometry of multifractal measures, Mathematische Nachrichten 204(1) (1999), 61-82.

DOI: https://doi.org/10.1002/mana.19992040105

[24] O. Frostman, Potential d'Équilibre et capacité des ensembles avec quelques applications á la théorie des functions, Meddel Lunds University Math. Sem. 3 (1935), 1-118.

[25] B. R. Hunt and V. Y. Kaloshin, How projections affect the dimension spectrum of fractal measures, Nonlinearity 10(5) (1997), 1031-1046.

DOI: https://doi.org/10.1088/0951-7715/10/5/002

[26] X. Hu and S. J. Taylor, Fractal properties of products and projections of measures in $\mathbb{R}^{d}$, Mathematical Proceedings of the Cambridge Philosophical Society 115(3) (1994), 527-544.

DOI: https://doi.org/10.1017/S0305004100072285

[27] E. Järvenpää, M. Järvenpää, F. Ledrappier and M. Leikas, One-dimensional families of projections, Nonlinearity 21(3) (2008), 453-464.

DOI: https://doi.org/10.1088/0951-7715/21/3/005

[28] E. Järvenpää, M. Järvenpää and T. Keleti, Hausdorff dimension and nondegenerate families of projections, The Journal of Geometric Analysis 24(4) (2014), 2020-2034.

DOI: https://doi.org/10.1007/s12220-013-9407-8

[29] R. Kaufman, On Hausdorff dimension of projections, Mathematika 15(2) (1968), 153-155.

DOI: https://doi.org/10.1112/S0025579300002503 
[30] J. M. Marstrand, Some fundamental geometrical properties of plane sets of fractional dimensions, Proceedings of the London Mathematical Society 4(1) (1954), 257-302.

DOI: https://doi.org/10.1112/plms/s3-4.1.257

[31] P. Mattila, The Geometry of Sets and Measures in Euclidean Spaces, Cambridge University Press, Cambridge, 1995.

[32] M. Menceur, A. Ben Mabrouk and K. Betina, The multifractal formalism for measures, review and extension to mixed cases, Analysis in Theory and Applications 32(4) (2016), 303-332.

DOI: https://doi.org/10.4208/ata.2016.v32.n4.1

[33] J. Li, L. Olsen and M. Wu, Bounds for the $L^{q}$-spectra of self-similar measures without any separation conditions, Journal of Mathematical Analysis and Applications 387(1) (2012), 77-89.

DOI: https://doi.org/10.1016/j.jmaa.2011.08.065

[34] T. C. O'Neil, The multifractal spectra of projected measures in Euclidean spaces, Chaos, Solitons \& Fractals 11(6) (2000), 901-921.

DOI: https://doi.org/10.1016/S0960-0779(98)00256-2

[35] L. Olsen, A multifractal formalism, Advances in Mathematics 116 (1995), 82-196.

DOI: https://doi.org/10.1006/aima.1995.1066

[36] L. Olsen, Mixed generalized dimensions of self-similar measures, Journal of Mathematical Analysis and Applications 306(2) (2005), 516-539.

DOI: https://doi.org/10.1016/j.jmaa.2004.12.022

[37] L. Olsen, Bounds for the $L^{q}$-spectra of a self-similar multifractal not satisfying the open set condition, Journal of Mathematical Analysis and Applications 355(1) (2009), 12-21.

DOI: https://doi.org/10.1016/j.jmaa.2009.01.037

[38] L. Olsen, On the inverse multifractal formalism, Glasgow Mathematical Journal 52(1) (2010), 179-194.

DOI: https://doi.org/10.1017/S0017089509990279

[39] B. Selmi, A note on the effect of projections on both measures and the generalization of $q$-dimension capacity, Problemy Analiza-Issues of Analysis 5(2) (2016), 38-51.

DOI: https://doi.org/10.15393/j3.art.2016.3290

[40] B. Selmi, Measure of relative multifractal exact dimensions, Advances and Applications in Mathematical Sciences 17(10) (2018), 629-643. 
[41] B. Selmi, Multifractal dimensions for projections of measures, Boletim da Sociedade Paranaense de Matemática (to appear).

[42] B. Selmi, On the strong regularity with the multifractal measures in a probability space, Analysis and Mathematical Physics 9(3) (2019), 1525-1534.

DOI: https://doi.org/10.1007/s13324-018-0261-5

[43] B. Selmi, On the effect of projections on the Billingsley dimensions, Asian-European Journal of Mathematics 13 (2020), 2050128/1-17.

DOI: https://doi.org/10.1142/S1793557120501284

[44] B. Selmi, On the projections of the multifractal packing dimension for $q>1$, Annali di Matematica Pura ed Applicata (to appear).

DOI: https://doi.org/10.1007/s10231-019-00929-7

[45] B. Selmi and N. Yu. Svetova, On the projections of mutual $L^{q, t}$-spectrum, Problemy Analiza-Issues of Analysis 6(1) (2017), 94-108.

DOI: https://doi.org/10.15393/j3.art.2017.4231

[46] N. Yu. Svetova, Mutual multifractal spectra II: Legendre and Hentschel-Procaccia spectra, and spectra defined for partitions, Tr. Petrozavodsk. Gos. Univ. Ser. Mat. 11 (2004), 47-56.

[47] N. Yu. Svetova, The property of convexity of mutual multifractal dimension, Tr. Petrozavodsk. Gos. Univ. Ser. Mat. 17 (2010), 15-24.

[48] N. Yu. Svetova, Mutual multifractal spectra I: Exact spectra, Tr. Petrozavodsk. Gos. Univ. Ser. Mat. 11 (2004), 41-46.

[49] N. Yu. Svetova, An estimate for exact mutual multifractal spectra, Tr. Petrozavodsk. Gos. Univ. Ser. Mat. 14 (2008), 59-66. 